\title{
The SLA (Second Law Analysis) in Convective Heat Transfer Processes
}

\author{
Heinz Herwig \\ Institute of Thermo-Fluid Dynamics, Hamburg University of Technology, Hamburg D-21073, Germany
}

Received: March 22, 2016 / Accepted: April 05, 2016 / Published: May 31, 2016.

\begin{abstract}
In all convective heat transfer situations, losses occur in the flow field (by dissipation) as well as in the temperature field (by conduction). Typically these losses are more or less quantified by the friction factor $f$ with respect to losses in the flow field, and the Nusselt number $\mathrm{Nu}$ for the heat transfer quality. Assessing the process of convective heat transfer as a whole, then becomes problematic because two different non-dimensional quantities, $f$ and $N u$, have to be combined somehow. From a thermodynamics point of view, there is a reasonable alternative: Since all losses become manifest in corresponding entropy generation rates, these rates are determined in the velocity as well as in the temperature field. Based on the integration of the entropy generation fields, an energy devaluation number is introduced. It basically determines how much of the so-called entropic potential of the energy involved in a convective heat transfer process is used within it. This approach is called SLA (second law analysis).
\end{abstract}

Key words: Second law analysis, energy devaluation number, entropic potential.

\section{Nomenclature}

$\begin{array}{lll}\dot{E}^{e} & \text { Exergy part of } \dot{E} & (\mathrm{~W}) \\ \dot{E}_{l}^{e} & \text { Exergy loss rate } & (\mathrm{W}) \\ N_{i} & \text { Exergy devaluation number } & (-) \\ \dot{S}_{i r r} & \text { Entropic potential } & (\mathrm{W} / \mathrm{K}) \\ \dot{S}_{D}^{\prime \prime \prime} & \begin{array}{l}\text { Local entropy generation rate by } \\ \text { dissipation }\end{array} & \left(\mathrm{W} /\left(\mathrm{m}^{3} \cdot \mathrm{K}\right)\right) \\ \dot{S}_{C}^{\prime \prime \prime} & \begin{array}{l}\text { Local entropy generation rate by } \\ \text { conduction }\end{array} & \left(\mathrm{W} /\left(\mathrm{m}^{3} \cdot \mathrm{K}\right)\right)\end{array}$

\section{Introduction}

Today, and even more in the near future, CFD (computational fluid dynamics) is a serious tool that helps to design and optimize complex convective heat transfer processes like that in a heat exchanger. An integral part of the optimization of such processes is the assessment of the whole process or certain aspects of it.

Typically the design and "optimization" is based on expert knowledge, with the help of certain transfer

Corresponding author: Heinz Herwig, professor, research fields: fluid mechanics, thermodynamics and heat transfer. coefficients like friction factors and Nusselt or Stanton numbers with respect to the device under consideration. This situation is fundamentally different, however, when there is access to the information about the detailed flow and temperature field provided by CFD solutions. Then not only data at certain in- or outlet cross sections are available (which are used to determine the above mentioned coefficients), but the flow and temperature fields as a whole are known. Assessment considerations can then be made with detailed information about exergy losses and entropy generation in the process under consideration. This way to approach complex convective heat transfer problems combines numerical results (CFD $\widehat{=}$ computational fluid dynamics) and entropy generation considerations (SLA $\widehat{=}$ second law analysis), and therefore is called the CFD-SLA approach. Both aspects will be explained in detail hereafter.

\section{The CFD-SLA Approach}

CFD can provide detailed results for the flow and temperature fields in convective heat transfer problems. 
They are subjected to certain approximations, however, since the solution domain is discretised, nonlinear differential equations are linearized and turbulent momentum and energy transfer has to be modelled. Nevertheless solutions can be found with a prescribed accuracy, at least in principle (assuming for example that turbulence modelling is adequate).

When convective heat transfer situations are considered there are two important aspects. The first one is about the amount of energy transferred while the second one refers to the quality by which this happens. Both aspects can and should be analysed separately. The quality aspect of the heat transfer can be assessed by SLA (second law considerations). They are a superior alternative to the conventional way of assessing the quality of convective heat transfer by a friction factor and a Stanton number, for example.

\section{Assessment Criteria}

\subsection{Conventional Assessment}

Convective heat transfer is characterized by dissipation in the flow field and unwanted temperature differences in the temperature field (which to a certain extent, however, are needed to facilitate the heat transfer). Often these losses are quantified by non-zero friction factors and non-infinite Stanton numbers, respectively. When the process as a whole should be assessed, then $f$ and $S t$ have to be combined in order to end up with a well-defined criterion by which the process or its modifications can be judged. When, for example, a process modification has been performed changing the coefficients from $f_{0}$ to $f$ and $S t_{0}$ to $S t$, respectively, an often used criterion is the coefficient of performance $\eta$, defined as [1]:

$$
\eta=\frac{S t / S t_{0}}{\left(f / f_{0}\right)^{1 / 3}}
$$

Here the question arises what a certain number $\eta$ means since $f$ and $S t$ are not two coefficients of the same kind. Note that, often $S t$ changes by a few percent while $f$ is taking values that are more than twice as high as before, for example, see Ref. [2] for more details.

\subsection{Alternative Assessment}

The problem with criteria like Eq. (1) in the previous subsection is the combination of two quantities which are not of the same kind in terms of their physical meaning. There is an alternative, however, when both aspects, i.e., losses in the flow and in the temperature field, are referred to their original physical cause: the entropy generation involved which can be expressed by the corresponding loss of exergy, see Ref. [3] for example. According to the so-called Gouy-Stodola theorem, see Ref. [4] for example, an exergy loss $E_{l}^{e}$ with respect to the exergy $E^{e}$ of an energy $E$ is linked to an irreversible increase of the entropy $S$ by:

$$
\mathrm{d} E_{l}^{e}=T_{\infty} \delta_{i r r} S
$$

with $T_{\infty}$ being the ambient temperature. Here the exergy part $E^{e}$ of an energy $E$ is that part of the energy which can be used as work in a process that ends at environmental conditions (also called: available work). Such exergy losses occur in the flow and the temperature fields alike, though by different mechanisms. Indicating entropy generation rates in the flow field by $\delta_{i r r}^{D} \dot{S}$ and that in the temperature field by $\delta_{i r r}^{C} \dot{S}$, the overall entropy generation rate in a convective heat transfer process is the sum of both and $\mathrm{d} \dot{E}_{l}^{e}$ becomes:

$$
\mathrm{d} \dot{E}_{l}^{e}=T_{\infty}\left(\delta_{i r r}^{D} \dot{S}+\delta_{i r r}^{C} \dot{S}\right)
$$

Since in all processes in which exergy counts, its losses should be as small as possible the optimisation criterion is obvious: $\dot{E}_{l}^{e}$ has to be minimized in a process. In order to achieve this, one has to determine the infinitesimal entropy generation rates $\delta_{i r r}^{D} \dot{S}$ as well as $\delta_{i r r}^{C} \dot{S}$ and integrate them with respect to the solution domain of the problem under consideration. 
Here the infinitesimal values of $\dot{S}$ are:

$$
\begin{aligned}
& \delta_{i r r}^{D} \dot{S}=\dot{S}_{D}^{\prime \prime \prime} \mathrm{d} V \\
& \delta_{i r r}^{C} \dot{S}=\dot{S}_{C}^{\prime \prime \prime} \mathrm{d} V
\end{aligned}
$$

with the local entropy generation rate $\dot{S}_{D}^{\prime \prime \prime}$ due to dissipation:

$$
\begin{aligned}
& \dot{S}_{D}^{\prime \prime \prime}=\frac{\mu}{T}\left[2\left[\left(\frac{\partial u}{\partial x}\right)^{2}+\left(\frac{\partial v}{\partial y}\right)^{2}+\left(\frac{\partial w}{\partial z}\right)^{2}\right]\right. \\
& \left.+\left(\frac{\partial u}{\partial y}+\frac{\partial v}{\partial x}\right)^{2}+\left(\frac{\partial u}{\partial z}+\frac{\partial w}{\partial x}\right)^{2}+\left(\frac{\partial v}{\partial z}+\frac{\partial w}{\partial y}\right)^{2}\right)
\end{aligned}
$$

and that due to heat conduction

$$
\dot{S}_{C}^{\prime \prime \prime}=\frac{k}{T^{2}}\left(\left(\frac{\partial T}{\partial x}\right)^{2}+\left(\frac{\partial T}{\partial y}\right)^{2}+\left(\frac{\partial T}{\partial z}\right)^{2}\right)
$$

The overall exergy loss rate in a finite volume $V$ then is (with the additional index $i$ explained later):

$$
\dot{E}_{l, i}^{e}=T_{\infty} \int\left(\dot{S}_{D}^{\prime \prime \prime}+\dot{S}_{C}^{\prime \prime \prime}\right) \mathrm{d} V_{i}=T_{\infty} \dot{S}_{i r r, i}
$$

with $\dot{S}_{i r r, i}$ as all the entropy generated by the process under consideration. In Eqs. (4)-(7), the local entropy generation rates have to be split into their mean and fluctuating parts when the flow is turbulent [5].

\subsubsection{The Entropic Potential}

Whenever a certain amount of energy is involved in a convective heat transfer process, this energy has a "history" and a subsequent "fate": It started as primary energy before it somehow became part of the process under consideration, and it finally will end as part of the internal energy of the ambient in the future (by subsequent processes not yet known).

In Fig. 1, this is illustrated for the energy rate $\dot{E}$ which originated as primary energy, i.e., as pure exergy and then in subsequent process, steps is gradually devaluated until it finally ends as part of the internal energy of the ambient, i.e., as pure anergy. On that way, two single process steps (out of many possible ones) are shown exemplary, one reversible and one irreversible energy transfer operation.

Convective heat transfer in a complex device is such a process step and it definitely will be irreversible. The degree of irreversibility is exactly what characterizes this process step by applying Eq. (8) in order to determine its exergy loss rate $\dot{E}_{l}^{e}$, now indicated as $\dot{E}_{l, i}^{e}$, because it is assumed to be process step $i$ in the overall "devaluation chain" illustrated in Fig. 1.

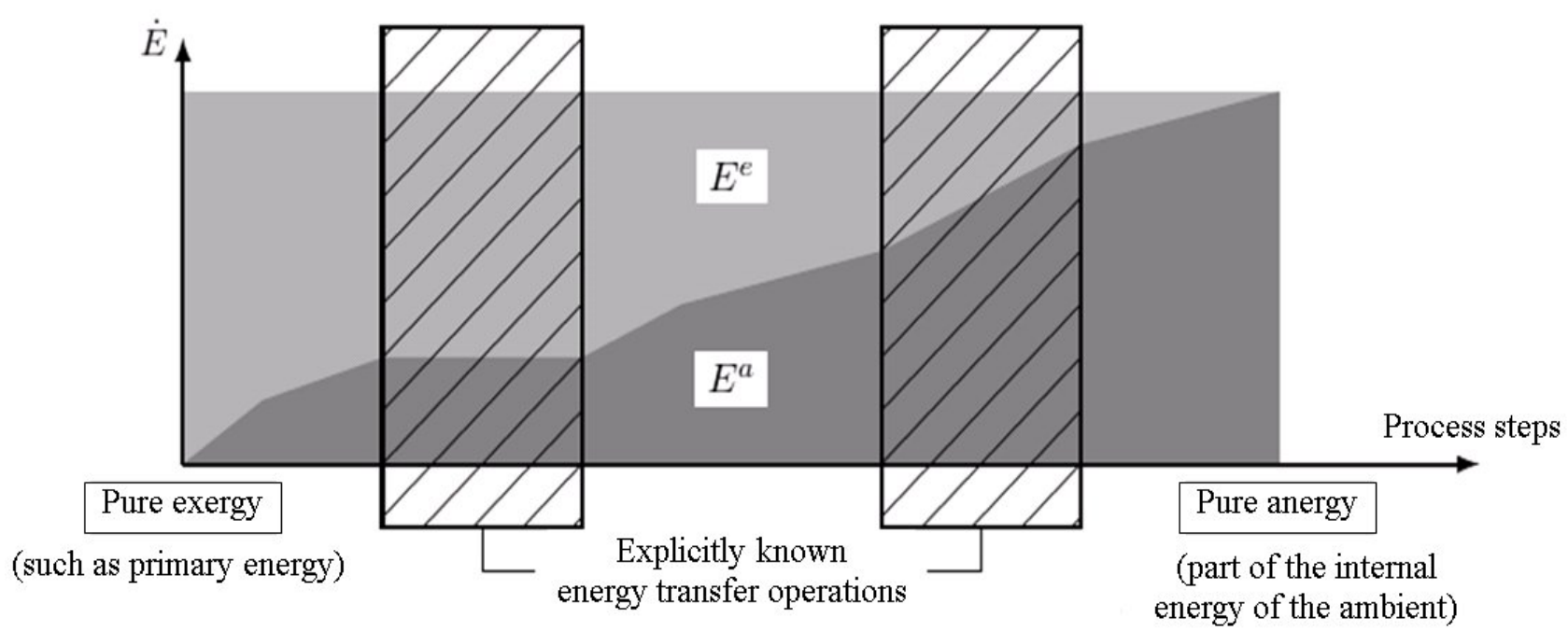

Fig. 1 Energy devaluation by consecutive energy transfer operations illustrated by the decrease of exergy during the energy transfer operations. Progress in process steps for finite energy rates. 
The overall devaluation is:

$$
\dot{E}_{l}^{e}=\sum_{i} \dot{E}_{l, i}^{e}=T_{\infty} \sum_{i} \dot{S}_{i r r, i}=T_{\infty} \dot{S}_{i r r}=\dot{E}
$$

where, $\dot{S}_{i r r}$ is the entropy generation rate by which the entropy of the ambient is increased when $\dot{E}$ becomes part of its internal energy. We call this quantity entropic potential [2] defined as:

$$
\dot{S}_{i r r}=\frac{\dot{E}}{T_{\infty}}
$$

and use it as a reference to assess the quality of a convective heat transfer process.

\subsubsection{The Entropy Devaluation Number}

The assessment of a specific process step which is an integral part of the "devaluation chain" with respect to the energy rate $\dot{E}$ involved in the process now is straight forward: The criterion is the amount of the entropic potential of $\dot{E}$ that is consumed by the process step $i$ under consideration. Therefore we define the dimensionless energy devaluation number for this step:

$$
N_{i} \equiv \frac{\dot{S}_{i r r, i}}{\dot{S}_{i r r}}=\frac{T_{\infty} \dot{S}_{i r r, i}}{\dot{E}}
$$

This number is always between 0 and 1 with both limits as:

$N_{i}=0$ : reversible energy transfer operation;

$N_{i}=1$ : energy transfer operation that completely devaluates the transferred energy rate.

When the overall devaluation of the energy rate $\dot{E}$ prior to the energy transfer operation, $i$ under consideration is given by the energy devaluation number $N$ and that after by $N_{\oplus}$ we have:

$$
N+N_{i}+N=1
$$

for the total devaluation chain. The energy devaluation number $N_{i}$ can be applied on very different process levels. In complex processes where $\dot{E}$ is split into several parts $\dot{E}_{i}$ and possibly rejoined again, $N_{i}$ can be calculated for appropriately defined sub-processes.

Examples in which $N_{i}$ is applied and compared to conventional assessment criteria can be found in Refs. [6-8].

\section{Conclusions}

Since nowadays complex convective heat transfer processes are more and more accessible to CFD solutions, a new strategy with respect to the assessment and optimization of these processes emerges. Within this approach, the quality of heat transfer can be assessed by referring to the second law of thermodynamics. The special and new aspect here is, that a certain heat transfer process should be incorporated into the overall picture of the energy transfer from the primary energy to the energy, being part of the ambient, by introducing the so-called entropic potential of that energy.

\section{References}

[1] Gee, D. L., and Webb, R. L. 1980. "Forced Convection Heat Transfer in Helically Rib-Roughened Tubes." Int. J. Heat Mass Transf. 23 (8): 1127-36.

[2] Herwig, H., and Wenterodt, T. 2013. "Second Law Analysis for Sustainable Heat and Energy Transfer: The Entropic Potential Concept." In Proceedings of the 5th International Conference on Applied Energy, ICEA 2013-343, 1-7.

[3] Bejan, A. 1995. Entropy Generation Minimization: The Method of Thermodynamic Optimization of Finite-Size Systems and Finite-Time Processes. New York: Taylor \& Francis.

[4] Moran, M. J., and Shapiro, H. N. 2007. Fundamentals of Thermodynamics. New York: John Wiley \& Sons.

[5] Kock, F., and Herwig, H. 2005. "Entropy Production Calculation for Turbulent Shear Flows and Their Implementation in CFD Codes." Int. J. Heat Fluid Flow 26 (4): 672-80.

[6] Redecker, C., and Herwig, H. 2014. "Calculating and Assessing Complex Convective Heat Transfer Problems: The CFD-SLA Approach." In Proceedings of the 15th International Heat Transfer Conference, IHTC 15-9184, $1-10$.

[7] Wenterodt, T., Redecker, C., and Herwig, H. 2015. "Second Law Analysis for Sustainable Heat and Energy Transfer: The Entropic Potential Concept." Applied Energy 139 (February): 376-83.

[8] Herwig, H., and Redecker, C. 2015. Heat Transfer and Entropy. Croatia: INTECH, 143-61. 